LHP: HOWELLS AND ROBERTSON

RHP: CHILDREN AS GAME DESIGNERS: NEW NARRATIVE OPPORTUNITIES

9

\title{
Children as Game Designers: New Narrative Opportunities
}

Cathrin Howells and Judy Robertson

\section{$<\mathrm{A}>$ Introduction}

"“The world narrated' is different to "the world depicted and displayed."

We are in a period of significant change in terms of writing; the supremacy of the written word for communication, which has held sway for centuries, is being challenged by multimodal forms of text, in particular the visual. Children are surrounded by images that convey meaning in often powerful ways, ways that can complement words but that can also go beyond words, carrying their own meanings, meanings that are more open to interpretation. These images are strongly rooted in the cultures of the young, often produced, received, and exchanged by electronic means, making them less accessible to the older, more traditionally (typographic) text-orientated generation. The meaning-making processes that children use when working with new texts may also be less familiar to adults. Texts are more 215 
fluid and interwoven, and boundaries and roles begin to blur as digital opportunities facilitate collaboration and textual change. Children often multitask with onscreen texts - several screens will be open at once, full of images as well as words, both moving and still; messaging flows alongside looking, reading, scrolling, hyperlinking, selecting, assembling, and creating new texts. The nature of composition has already changed from almost exclusively a process largely dependent on generating new typographic text from scratch, often emulating recognizable forms and formats, to the widespread practice of synthesis, fashioning text from words, images, and sounds from a variety of sources in a fluid and playful fashion. As new technologies emerge, so do new possibilities for communication; new composition practices and products evolve and our understandings of what constitutes 'text' must grow. A challenge for educators is to keep pace with learners as they increasingly draw on their own social (electronic) contexts for 'textual' inspiration.

Such challenges have been noted before. Kress (2000: 183) states that "we...find ourselves singularly ill-equipped in the new landscape of communication" but Pahl and Rowsell (2005: 41) warn that "We cannot afford to ignore the communicational landscape our students find themselves in. If we harness it and tie it to literacy learning, its potential is huge... It is our challenge to capture its flows and ebbs, and to develop a dialogue with our students..." And Jewitt (2008: 9) urges that it is necessary to reconceptualize literacy within education in the light of a strong "turn to the visual." To some degree, the new Curriculum for Excellence in Scotland (Education Scotland, 2010a) has responded to this twenty-first-century challenge in the literacy and English guidelines: "The definition of 'texts' also needs to be broad and future proof. Within the Curriculum for Excellence, a text is the medium through which 
ideas, experiences, opinions and information can be communicated" (Education Scotland, 2010a: 4). This is echoed in the technology curriculum (Education Scotland, 2010b), which specifies that children should become confident users of technology now and in the future, becoming informed users and producers; the outcomes expect the complex new media authoring task of game design to be part of the classroom experience at upper primary and lower secondary levels. These values to provide a relevant curriculum are also enshrined in the aims and values of the national curriculum in England (DfE, 2011).

Given these forward-looking aims, and the challenges that teachers face in implementing them in everyday classroom life, we argue that we need to better understand the literacy opportunities afforded by new media authoring environments. Further, we need to identify successful strategies that learners may use to create communicative artifacts that are of value to themselves, their teachers, and their peers. Pahl and Rowsell (2005) comment that in fact where new media is concerned, teachers should be prepared to learn from children, who may have different ways of knowing.

Our contribution in this chapter is to investigate one new media example: fictional, narrative, interactive, immersive 3D multimodal game texts created through computer game authoring. This is a challenging medium that offers learners powerful new opportunities to explore 3D spatial design as a method of telling stories. We make particular reference to how aspects of this medium extend Kress's notion of reading pathways, the routes that a reader will take through any text, be it conventional or multimodal (Kress \& van Leeuwen, 2006). We also consider the role of new 'writing' elements we call 'story locations' and 'narrative vehicles.' This medium challenges learners by asking them to develop new techniques for conveying 
the story to the reader using largely the visual and spatial design of the storyworld, with typographic text playing only one part in the process of creating plot elements. The order in which players then encounter these plot elements depends on how they choose to explore the 3D spatial world. The choices players make will also alter their experience of the story, and the author must anticipate this.

As with any rapidly developing medium, it is hard to find an accessible source of practical knowledge on the authoring process that could apply to young learners. Indeed, Marsh and Singleton (2009) note that we are only just beginning to understand what virtual worlds of this kind might mean for literacy practices. We take advantage of the fact that young learners may in fact be more experienced consumers of new media than adults; our approach here is to analyze some strategies used by learners who have used game authoring tools to tell stories during a six-week classroom-based field study. Based on examination of the authoring process used by learners who appear to have a natural talent for making games, compared with those learners who were less successful, we can identify ways of working that may be useful to other learners in the future.

\section{$<$ A $>$ Creativity in Writing}

Grainger et al. (2005) stipulate that literacy learning should be highly motivating and interactive and build on the social context of learners. Sharples (1999) conceptualizes writing as a creative design process, while Kress (2003) talks of the concept of competence in language use giving way to that of interested design. He suggests an approach to text creation that encourages and normalizes 'design' of text and views innovation and creativity as the norm that will equip learners and their teachers to respond to constant changes in media. 218 
Kress considers creativity an automatic consequence of a design-based approach to message creation: "Creativity becomes normal and unremarkable in every instance of sign-making" (Kress, 2003: 169). Grainger et al. agree that creativity has to be central to the writing process, facilitating not only children's growth as writers but also their self-esteem. Kress foresees a new and more prominent role for imagination in literacy practices. He suggests that competence in one mode, with little place for imagination, will be replaced by the much more demanding tasks of "selection, arrangement and transformation, involving many modes, in always new environments, with their always changing demands.” (Kress, 2003: 171). Grainger et al. (2005: 23) also stress the importance of imaginative and creative contexts for writing: “A creative context implies an environment of possibility, which offers choice and encourages children to experiment with ideas, take intellectual risks and find innovative ways forward with speech and writing. Such an environment is both supportive and challenging although is not without frames of reference."

\section{$<$ A $>$ New Forms of Text}

Alphabetic text is rapidly becoming less dominant under the increasing influence of both the still and the moving image and the multimodal texts (employing pictures, sound, movement, color, texture) that are becoming the norm (Kress, 2003; Lankshear \& Knobel, 2006; Jewitt, 2008). In such texts, each mode influences the other and the result is often multilayered in meaning and non-linear in nature with the screen rather than the page providing the canvas. Such texts are certainly suitable vehicles for Kress's idea of 'interested design' and the 'future-proof' twenty-first-century texts of A Curriculum for Excellence (Education Scotland, 
2010a). Both the reading and the writing of multimodal texts differ from the requirements for conventional texts.

Alphabetic, conventional text has a temporal, sequential logic, whereas the logic of image is spatial and simultaneous. In multimodal texts, the sequential and the spatial exist side by side and "the images often communicate different things from the words. And the combination of the two modes communicates things that neither of the modes does separately." (Gee, 2003:

Field Code Changed 14). No one mode carries the full meaning and children will experiment to create "a 'multimodally' conceived text, a semiotic play in which each mode, the verbal and the visual, is given a defined and equal role to play.” (Kress \& Van Leeuwen, 2006: 113)

Kress stresses the importance of 'reading pathways,' the routes that a reader will take through any text, be it conventional or multimodal. These pathways are signposted and scaffolded to varying degrees, ranging from the provision of a firm hand to guide the reader (typical of conventional texts) to a few hints and suggestions (the semi-linear text) to texts where no one pathway is any more plausible than any other . In the medium of 3D computer games, on 
which this paper focuses, the reading pathways become far more complex and unpredictable than in a $2 \mathrm{D}$ text, not least because the reader (player) has the facility to take a 360 -degree view of the world he or she is exploring, and is free to make many open-ended choices.

\section{$<\mathrm{A}>$ The Computer Game as Text}

Compared with other multimodal texts, computer games offer added complexity for both designer and player, ${ }^{1}$ including the challenge that the player (anticipated by the designer) can move around inside the world of the text and experience it from more than one visual, spatial, and textual perspective. Other studies, including several reported in this book, have explored the potential literacy development benefits for players who take part in virtual worlds; this chapter moves beyond this to focus on the literacy opportunities for learners when they design their own games. From this point in the text we use the term 'designer' to refer to the learner who is creating a game, a role similar to that of the writer in conventional texts. The 
term 'player' is used to describe the role of the person who takes part in the game produced by the designer. This role encompasses the role of the reader of a conventional text.

Further, it is perhaps useful to mention that while previous researchers have made much of the conflict between narrative and gameplay, this theoretical debate does not have much bearing on the current discussion, as we are deliberately focusing on games authoring as a way of developing storytelling skills at the expense of developing skills in designing game mechanics.

Both game design and the playing of the finished game offer a highly interactive, spatial, and open-ended creative experience. The designer of the computer game must consider the creation and appearance of the world, the telling of the story, and the management of the player; the concept of salience is relevant here. Personal and cultural preferences come in to play as children decide where and how to place the key elements of their design and consider how to attract the player's attention to them and so to possible pathways through the $3 \mathrm{D}$ text. However, what might be salient to the designer is by no means guaranteed to carry the same weight of meaning for the player. Kress's more recent work acknowledges the need to reconsider the notion of reading pathways in its traditional form for digital texts, introducing the concept of 'reading as design': “The reader's interest determines how she or he will engage with the page and establishes the order in which its elements are 'read.' It is the reader's interest which provides the design of this page in that respect” (Kress, 2010: 175). These texts, which are decidedly complex in nature, create new challenges for both designer and player, not to mention the teaching profession; they provide the opportunity to see new 
behaviors and techniques at work, including 'reading as design,' and make us think again about what 'creative writing' can mean.

\section{$<\mathrm{A}>$ Interactive Game Authoring}

\section{$<$ B $>$ Software for Game Creation}

Adventure Author is a computer game authoring tool for children aged 10-14, developed at Heriot-Watt University with the goals of promoting literacy and creativity. It is based on the commercially available Neverwinter Nights 2 (NWN2) game authoring software, which provides a toolset allowing children to design an interactive game world and people it with creatures and plot items ([INSERT FIGURE 9.1 HERE]

Figure 9.1 Area design within the NWN2 Toolset.

\section{[INSERT FIGURE 9.1 HERE]}

Figure 9.1 Area design within the NWN2 Toolset.

\section{[INSERT FIGURE 9.1 HERE]}

Figure 9.1 Area design within the NWN2 Toolset.

The software also offers children the opportunity to create dialogue via the Conversation

Writer (see Error! Reference source not found.), and children can add story text to objects 
such as journals and signposts to help the narrative unfold and bring characters to life (Error! Reference source not found.).

\section{[INSERT FIGURE 9.2 HERE]}

Figure 9.2 A cloak with added text gives further information to the player.

These games are complex, interactive, immersive texts combining typographic text, image, sound, and 3D spatial opportunities, and children have to go beyond the demands of conventional writing in order to create them.

\section{$<$ B $>$ Game Authoring in the Classroom}

In order to explore the strategies young learners might use to tackle the complex task of game authoring, we undertook a naturalistic field study with the help of an enthusiastic teacher. The field study was conducted with a class of 25 pupils (aged 11-12) in a state-funded primary school in East Lothian, Scotland. The class teacher wished to try the Adventure Author software to support a cross-curricular project in fantasy literature themed around the novel The Hobbit by J. R. R. Tolkien. She chose to spend most of the class time for a six-week period on this project, although there were also regular timetabled slots for mathematics and P.E. By examining the strategies used by successful designers, we aimed to identify useful approaches that could be of assistance to other learners or teachers in the future.

\section{$<$ B>Dialogue: Choices, Actions, and Conditions}

Dialogue is the main means of taking the game forward in any chronological or sequential way - and given there is no descriptive text or narration the dialogue has to work harder than 
in a conventional written story. Dialogue also has a crucial part to play in terms of characters: children introduce their characters and their roles via dialogue and bring them to life through the words that they say and the actions ascribed to them. The Conversation Writer in Adventure Author makes it easy to branch a conversation (Figure 9.1) in order to give the player choices and to add actions or conditions to a line of dialogue, to set up rewards, attacks, and quests. Creating a choice for the player is relatively easy, but the designer must understand the tree-like structure that results from the choices. Sometimes the branching option helps to bring out the personality or feelings of a character. We illustrate these design choices through focusing on specific designers' decisions.

\section{[INSERT FIGURE 9.3 HERE]}

Figure 9.1 The structure of branching dialogue in the Conversation Writer.

Stewart (Error! Reference source not found.) experimented with three dialogue choices for the player. Option one: the player does not agree to a character's request; option two: the player agrees to help; and option three: the player considers the request, but needs to be persuaded to continue. The player can explore all three, with attendant consequences.

\section{[INSERT FIGURE 9.4 HERE]}

Figure 9.4 A dialogue with three choices; note diagrammatic representation bottom right.

Setting up actions in a conversation (Figure 9.2) was popular.

[INSERT FIGURE 9.5 HERE]

Figure 9.2 Actions (in bold) added to dialogue in the Conversation Writer. 
Tania confidently used a mixture of choices and actions with some witty dialogue to create lighthearted banter between the player and a pixie. The pixie warns, "Ah, you may choose to look through a rose tinted monocle or be wary and safe. Do you REALLY trust me?" The player can either agree (receiving 50 gold pieces), or disagree and kill the pixie. If the player does trust the pixie, it ultimately becomes a bad-tempered ally, resulting in the frustrated player's outburst, "If you don't shut up, I'm going to clip your wings and take away your magic!"

The children enjoyed giving the player rewards, giving the player an ally, or specifying that a character should attack a player. They seemed to relish the opportunity to program the behavior of the game.

It is also possible in the Conversation Writer to set up conditions under which particular dialogue will appear to the player. The designer is essentially specifying a series of IF-THEN rules, such as "IF the player is carrying item x THEN say dialogue line Y." Morag used a condition to set up a quest in which the player must find a secret book before she can help defeat the dragons that threaten the villagers (Figure 9.3).

\section{[INSERT FIGURE 9.6 HERE]}

Figure 9.3 Morag sets conditions.

Writing interactive dialogue is challenging at a number of levels. The learner must first master the technical and logical aspects of setting up choices, actions, and consequences.

They must also think about the vocabulary for the conversation to make it genre-appropriate, as well as techniques for portraying personality or moods. Lastly, they must consider how 
this dialogue fits in with the plot, and particularly how the dialogue branches can lead to different plot outcomes. This is both cognitively and imaginatively demanding; most learners of this age require support with this task.

\section{$<$ B $>$ Story Locations}

In the absence of conventional narrative or descriptive text, the designer uses the multimedia tools: visually, to create an exciting environment, and spatially, to draw the player in a particular direction. Many children take pains to make the setting fit their storyline, and create what we have called story locations, i.e., small scenes within the wider game setting (a cave with a glowing light at the door; a blacksmith's forge complete with the sound of hammering): significant places where significant events occur. These events, perhaps a conversation, or the acquisition of an item, a trap, or a transition to another part of the game, move the story forward. They happen in a significant location. Story locations and their associated events might be seen as the equivalent of chapters or episodes.

Stewart has given a lot of thought to such locations in his game (see Figure 9.4Figure 9.4), creating interesting settings in each quarter of the area grid.

\section{[INSERT FIGURE 9.7 HERE]}

Figure 9.4 Stewart's game has four distinct story locations with effective landscaping. The black dragon, critical to the quest, is placed in a hollow on a hillside, surrounded by special effects, drawing the player's attention and creating a dramatic arena for some important dialogue. Diagonally opposite, the glow spider, who will help the player, nestles in a curve of a stream with a waterfall as a backdrop - but beware the traps either side. An 
amulet, the object of the quest, is hidden bottom right, beside a rocky cave, guarded by goblins and trolls; this entire location is partially screened by hills and a building, but lighting hints at its presence. Diagonally opposite this, top left, across a stream and beside a forest, is the hillside location for the evil gargoyle that has to be defeated. Stewart seems to have a clear idea of what will happen in his game, where and when; in each of these locations the player receives new information or something significant occurs, and he has taken care to add supporting detail and interest.

Angus is less successful in harnessing the multimedia tools to create story locations (see Error! Reference source not found.). His area is packed with buildings and monsters and soldiers; it lacks coherence and suggests more random experimentation. For example, the bridge, which may seem inviting, leads nowhere, and the ships around the edge of the area are not in water. It may be that his storyline was less clear in his mind, or that he had less knowledge about how to 'chunk' his story into different locations across space and time.

\section{[INSERT FIGURE 9.8 HERE]}

Figure 9.8 Screenshot of Angus' game showing a less coherent area design.

The creation of effective (and genre-appropriate) story locations within the game world to support an unfolding story would seem to require a degree of sophistication. The designer needs a clear idea of the overall plan, a linking of visual, spatial, and temporal thinking, and an understanding of how the player might operate. It may be that additional help is required to map the game world in advance, with discussions about the way the story will move across this map from event to event. 


\section{$<$ B $>$ Narrative Vehicles}

Children can choose particular items (such as books, furniture, or other props) to play a significant role. They might become the focal point for a pathway through the game - e.g., to be collected as part of a quest, and narrative text can be added. For example, the designer might make a magic book containing information to be revealed only at a certain point in the game under certain conditions. When an item has been given this sort of role, we have called it a narrative vehicle, i.e., it helps to carry the story forward in some significant way.

Morag successfully uses narrative vehicles to contribute to her storyline. Her character Neeshka charges the player with a quest to find the potion of Lion's Heart, explaining that she feels weak and shy without it. When the player successfully delivers it to Neeshka, she is rewarded with both gold coins and storyline advancement, as Neeshka is now brave enough to help fight the dragons.

Kirsty also experiments with using narrative vehicles, but perhaps less successfully. She has written text for a series of genre-appropriate, carefully named objects: the Eidilon Temple Book, an invisibility potion, the village amethyst, the Starter Staff, and the Eidilon Crystal. The text in each gives tantalizing hints of the plot line - e.g., "A long time ago the people of Eidilon village were enemies, now this one gem is what brings peace over the land."

Although the amethyst to which this text belongs is placed on a route that the player is likely to take through the game, it is not built into the storyline thereafter.

It seems that Kirsty is good at imagining potential plotlines for the fantasy genre but has not quite worked out how to build them into a spatial story environment. The player might reasonably expect to interact with the narrative vehicles in order to influence the plot, but 
Kirsty has not used the procedural aspects of the game authoring tool to program in these possibilities.

Children will need support to handle narrative vehicles effectively, balancing interesting textual content with careful thought as to how the player will find and interact with these important objects - e.g., by setting conditions.

\section{$<$ B $>$ Reading Pathways: Multimedia}

Reading pathways, the routes through the game, can be indicated using the multimedia features of the software. The most successful children created pathway possibilities that were convincing, attractive, and supportive of their player.

\section{[INSERT FIGURE 9.9 HERE]}

Figure 9.9 Red arrows show likely pathways created by Robert's use of multimedia tools; blue arrows show possible routes where player will quickly come to a dead end.

Robert has given a lot of thought to supporting his player, creating a visually appealing and convincing world, but one where the possibility of losing the trail is minimized. Robert's intended pathway (Error! Reference source not found.) flows through a narrow strip of low ground at the foot of the area, and any interesting distractions (e.g., buildings in the distance) soon prove to be inaccessible, minimizing player frustration and helping to keep them en route to the main objective - crossing the Misty Mountains (suggested by the high ground behind). He carefully draws his player towards the smithy with the sounds of the blacksmith's forge, helping to ensure they meet the narratively important dwarf, and then draws them on to the rising ground behind the smithy by placing a decorated building and a key character where they will be noticed. As the player climbs further, his or her eye will be drawn to a distinctive gate, indicating a possible transition to Robert's next level. 
Although Kirsty (Figure 9.5Figure 9.5) has clear visual pathways in her design, they are not

Formatted: Font: Not Bold

effectively incorporated into her game and the player may actually have more success if he or she ignores them. She has a clear idea of how the story unfolds in her own mind, and the paths are more visually dominant than in Robert's design, but she is less successful in guiding the player. Kirsty uses cobbled paths, signs, and 'meet and greet' characters to try to direct the player, who has to find the red dragon to understand the purpose of the game. There are narrative vehicles containing excellent story information lying on the cobbled paths, but no clues as to how to find or use them. In addition, she fails to set conditions on significant lines of dialogue that would allow her greater control over sequencing the spatial pathways. This means her player is at risk of missing the key facts of the game, and is certainly unlikely to come across them in the correct order.

\section{[INSERT FIGURE 9.10 HERE]}

Figure 9.5 Kirsty's game. Solid red lines show the pathway her narrative seems to require; broken red lines show some of the other routes the player would be just as likely to take.

\section{$<$ B $>$ Reading Pathways: Textual}

The children used alphabetic text to indicate pathways through their game, giving instructions and guidance to the player via dialogue, narrative vehicles, and floating text overlays. The most successful games effectively combine both textual and digital support to guide the player through the $3 \mathrm{D}$, interactive, immersive world.

Stewart's game (whose layout offers no overt visual paths at all - see Figure 9.4Figure 9.4) uses textual cues to indicate pathways to his carefully crafted story locations. Unlike Kirsty, 231 
he has harnessed the condition function of the Conversation Writer, allowing him to control the progress of his player, and his clear story locations give a focal point to aim for. The result is a spiral route through the world of his game (see Error! Reference source not found.) with conditions governing dialogue with the black dragon (location 5) such that the player must return via locations 3 and 4 with an amulet in order to gain additional information to complete the quest (6).

\section{[INSERT FIGURE 9.11 HERE]}

Figure 9.11 Stewart's area design, with likely reading pathways indicated by red arrows.

Here is a typical example of Stewart's directional dialogue, between the player and the glow spider as he explains he must help the black dragon:

Spider: You will need his amulet, but the goblins took it and hid it somewhere in a cave in this land.

Player: I will find it.

If Stewart had not given such careful thought to his game world, this dialogue would be too open-ended, but he has seeded enough visual clues to ensure an alert player will head off in the right direction - the cavd is tantalizingly visible beyond the southern hills.

By contrast, Angus's only ex 5 le of dialogue is vague (Error! Reference source not found.), offering little by way of pathways or purpose in what we have already seen is a very cluttered game world (Error! Reference source not found.).

[INSERT FIGURE 9.12 HERE]

Figure 9.12 Angus' instructions are vague.

232 
Hester creates floating text to appear in the game, via the Display Message action in Conversation Writer, which tells the player, "Go and search around and see what everyone is up to." This suggests a random or open-ended series of pathways, whereas Sandy chooses to display the more specific message, "Go to the woods." Kirsty's potion of invisibility gives the player an even more specific direction by saying, "Give this potion to Yuki of the winter wolf clan for a reward!"

As a game designer, it is not enough to use text in order to set up pathways for the player; that text also has to make successful partnerships with the 3D spatial, immersive, and interactive aspects of the toolset to create effective multimedia pathways; the challenge for the children is how to combine all these features so that each plays an effective part in guiding the player through their world.

\section{$<$ A $>$ Discussion}

While considering these games, some points of interest emerged. Firstly, it became clear that storytelling skills from traditional writing tasks did not always transfer into the game authoring medium. Games with a lot of dialogue and narrative vehicle text are often not successful, particularly because players can get impatient with reading onscreen text during gameplay. Designers are sometimes disappointed to see a player scroll through their carefully crafted text without reading it. From the player's perspective, too much text slows down the action of the game and can reduce his or her sense of participation. Teachers can support learners by helping to identify sections of the game that are too text-heavy and by assisting dialogue editing. The writer's maxim "show, don't tell” is particularly relevant here. 
Secondly, there are some new techniques to be mastered that go beyond traditional textual storytelling techniques - developing story locations, using narrative vehicles, managing pathways in a highly complex narrative environment, and making effective use of dialogue in this context. These are complementary elements that form a significant part of the storytelling and take it beyond conventional narrative. As designers, children have to make decisions, textually, visually, spatially, about how overt they want their reading pathways to be, how they want to use narrative vehicles, where and how to create and link their story locations, and how they will harness speech. These design decisions are a fascinating mix of modal meanings that need to be carefully combined; in order to facilitate this, teachers will need to become much more familiar with the possibilities offered by this form of storytelling.

Thirdly, learners who did not have much experience of playing computer games sometimes underestimated the importance of the 3D spatial aspect of the medium, or perhaps were unaware of how to use it to tell a story. A possible reason for this is that they did not visualize the game as the player would see it from beginning to end. The toolset defaults to a top-down view of the landscape that emphasizes the area as a whole rather than what the player will see minute by minute. When playing the game, the player sees the world from the point of view of the character rather than as an overview. To understand the player's experience it is necessary for the designer to regularly switch points of view by testing the game. In testing the game, the designer is explicitly trying out reading pathways in order to experience his or her work from the player's perspective. Anticipating the reader's needs is important in conventional writing tasks, but it can be difficult to encourage young authors to explicitly 
switch between the role of author and reader in order to improve a text; Adventure Author encourages switching between the roles of designer and player, and the process of checking and editing is much more immediate. To help learners with less experience of games, teachers could encourage learners to play example games before they work on their own games, and to regularly and systematically test their games throughout the process, ensuring that as author/designer they take the opportunity to change perspective and explore the needs of their reader/player.

\section{$<\mathrm{A}>$ Conclusions}

Creating a 3D, interactive, immersive game provides new opportunities for authors. Dialogue has to work in new ways and be married with logical thinking and spatial awareness to manage a quest structure; the narrative vehicle and the story location are new concepts whose potential needs to be explored and understood; and the many ways of creating and combining reading pathways, the key to following the story, have to be mastered. Computer game authoring also allows children's own cultures and experiences to have a place in the classroom and provides an opportunity for both teachers and children to be creative in their approaches.

The challenges for the learner are many when designing stories in this medium. They must consider: How do I build a story spatially? How can I anticipate what my reader, the player, might do? Under what circumstances should I try to tell the story through words and when should I use visual design? The challenges for the educator involve supporting their learners in the creation of these new forms of text and the literacy behaviors that go with them. If educators are to acquire knowledge of successful new media authoring strategies, and assist 235 
their pupils in effectively expressing themselves, perhaps they need to become multimodal designers in their own right. They certainly need to prepare the structured teaching interventions, exemplars, assessment schemes, and software scaffolding to provide the welljudged frames of reference recommended in Jewitt's literature review (Jewitt, 2008). Much of this is new and changing territory, which may take teachers beyond their comfort zones and challenge their understandings of what is meant by 'text' and 'literacy'; and indeed there are elements of risk attached as educators find their way forward. But the evident engagement, motivation, and wealth of learning opportunities afforded by computer game authoring increases the imperative on the education world to meet these challenges. Kress (2003: 175) warned: "The young are teaching themselves because the old cannot or will not."

This paper begins to bridge the gap between the understandings of the young, who hold the answers for the future, and the understandings of the old, who have the answers from the past.

\section{$<$ A $>$ References}

Curriculum Review Group (2008). Cover paper for draft experiences and outcomes in Technologies. Glasgow: Learning Teaching Scotland.

Curriculum Review Group (2007). Cover paper for draft experiences and outcomes in Literacy and English. Glasgow: Learning Teaching Scotland.Retrieved May 18 2012 from Formatted: Superscript http://www.ltscotland.org.uk/curriculumforexcellence/outcomes/technologies/index.asp. DfE (2011). The Curriculum: Aims Values and Purposes. Retrieved January 13, 2012 from http://www.education.gov.uk/schools/teachingandlearning/curriculum/b00199676/aimsvalues-and-purposes. 
Education Scotland (2010a). Curriculum for Excellence: Literacy Across Learning.

Principles and Practice. Retrieved January 13, 2012 from

http://www.ltscotland.org.uk/learningteachingandassessment/learningacrossthecurriculum/res ponsibilityofall/literacy/principlesandpractice/index.asp .

Education Scotland (2010b). Curriculum for Excellence: Technology. Principles and

Practice. Retrieved January 13, 2012 from

http://www.ltscotland.org.uk/learningteachingandassessment/curriculumareas/technologies/pr inciplesandpractice/index.asp.

Gee, J. P. (2003). What Video Games Have to Teach Us about Learning and Literacy. New York: Palgrave Macmillan.

Good, J. \& Robertson, J. (2006). CARSS: A framework for learner-centred design with children. International Journal of Artificial Intelligence in Education 16(4): 381-413.

Grainger, T., Goouch, K., \& A. Lambirth (2005). Creativity and Writing: Developing Voice and Verve in the Classroom. Abingdon: Routledge.

Jewitt, C. (2008). The visual in learning and creativity: A review of the literature. A report for Creative Partnerships. London: Institute of Education, University of London.

Juul, J. (2001). Games telling stories? Games Studies 1(1). Retrieved January 13, 2012.

http://www.gamestudies.org/0101/juul-gts/.

Lankshear, C., \& Knobel, M. (2006). New Literacies:Everyday Practices and Classroom Learning. Open University Proess McGraw-Hill.

Kress, G. (2003). Literacy in the New Media Age. London: Routledge. 
Kress, G. (2000). Multimodality. In B. Cope \& M. Kalantzis (eds.), Multiliteracies: Literacy Learning and the Design of Social Futures. London: Routledge.

Kress, G. \& van Leeuwen, T. (2006). Reading Images: The Grammar of Visual Design. London: Routledge.

Kress, G. (2010). Multimodality: A social semiotic approach to contemporary communications. London: Routledge. Formatted: Normal (Web), Indent: Left: $0 \mathrm{~cm}$, Hanging: $0.85 \mathrm{~cm}$

Marsh, J. \& Singleton, C. (2009). Literacy and technology: Questions of relationship

[Editorial]. Journal of Research in Reading 32(1): 1-5.

Merchant, G. (2009). Literacy in virtual worlds. Journal of Research in Reading 32(1): 38 56.

Murray, J. (1997). Hamlet on the Holodeck: The Future of Narrative in Cyberspace. New York: Free Press.

Pahl, K. \& Rowsell, J. (2005). Literacy and Education: Understanding the New Literacy Studies in the Classroom. London: Paul Chapman.

Robertson, J. \& Good, J. (2003). Using a collaborative virtual role-play environment to foster characterisation in stories. Journal of Interactive Learning Research 14(1): 5-29.

Robertson, J. \& Howells, C. (2008). Computer game design: Opportunities for successful learning. Computers and Education 50(2): 559-578.

Sharples, M. (1999). How We Write: Writing as Creative Design. London: Routledge. 